\title{
Is the SARS-COV-2 Carcinogenic?
}

\section{Sreelekha Ray*}

Consultant, Head Neck Oncosurgery, Deenanath Mangeshkar Hospital and Research Center, Pune, Maharashtra, India

*Corresponding Author: Sreelekha Ray, Consultant, Head Neck Oncosurgery, Deenanath Mangeshkar Hospital and Research Center, Pune, Maharashtra, India.
Received: June 04, 2021

Published: July 01, 2021

(C) All rights are reserved by Sreelekha Ray.

Keywords: SARS-CoV-2; circRNA; Cancer

\section{Abbreviations}

SRS-CoV-2: Severe Acute Respiratory Syndrome Coronavirus 2; circRNA: Circular RNA; MERS-CoV: Middle East Respiratory Syndrome Coronavirus; Pre-mRNA: Precursor Messenger RNA; miRNA: MicroRNA; ${ }^{6}$ A: N6-methyladenosine; IG-1: Retinoic acid-inducible Gene I; COVID-19: Corona Virus Disease 2019; NK: Natural Killer; $\mathrm{CD}^{8+}$ : Cluster of Differentiation $8 ; \mathrm{CD}^{4+}$ : Cluster of Differentiation 4; PD-1: Programmed Death-1; PDL-1: Programmed Death Ligand-1; IFN-1: Interferon-1; AR: Androgen Receptor; pRB: Retinoblastoma Protein

\section{Can SARS-CoV-2 cause cancer?}

The SARS-CoV-2-induced pandemic has disproportionately affected patients with pre-existing malignancies. But recent researchers [1] have postulated that the virus may have malignant potential via circRNAs, while identifying and characterising them in MERS-CoV and SARS-CoV-1/2. With a sense of urgency, we need to understand the connection between the SARS-CoV-2 and its impact on cancer. If the connection(s) can be proved, the pandemic will have more long-lasting, far-reaching deleterious consequences than previously thought.

The etiological relationship between circRNAs and cancers has been discussed in context of known oncogenic viruses, and whether circRNAs can be potential biomarkers for their prognosis and diagnosis. But direct evidence linking with SARS-CoV-2-encodedcircRNA with cancer initiation, progression, metastasis, relapse, and chemotherapy resistance is lacking, although there is compelling circumstantial evidence.

CircRNA is the new kid on the block

CircRNAs when first discovered in late 70s were regarded as products of incorrect splicing of pre-mRNAs. But since 2013, they have been recognised as ubiquitous, single-stranded, stable, highly conserved, cell- and tissue-specific molecules, indicating that they are not erroneous or random by-products in the RNA network [2].

Earlier thought to be non-coding sequences, circRNAs have now been found to encode proteins, interact with other proteins, participate in protein complex scaffolding, parental gene modulation, RNA-protein interactions and miRNA sponge [3]. CircRNAs function as miRNA sponges by removing them from their site of action. This can contribute to cancer progression by sponging tumoursuppressive miRNAs, or cancer suppression by sponging off oncogenic miRNAs. CircRNAs have thus been implicated in several of the hallmarks of cancer [4].

Cross talk between viral circRNA and immune system

In infections with oncogenic viruses, the expression of host circRNAs is dysregulated in virus-infected cells, and the viruses can use these molecules for their own purposes. In addition, some viral genes are able to produce viral circRNAs by a back-splicing mechanism [5]. The viral circRNA on entering the cytoplasm, is recognises the modified $\mathrm{m}^{6} \mathrm{~A}$ protein as non-self by the novel RIG- 
1 mechanism, which stimulates innate immunity in the competent immune system [6]. Patients with severe COVID-19 disease exhibit lymphocytopenia and suffer from T-cell exhaustion, with reduced levels of circulating NK cells, $\mathrm{CD}^{+} \mathrm{T}$ cells, and to a lesser extent, $\mathrm{CD}^{+}$cells [7]. In the tumour microenvironment, the effector functions of these cells are impaired by the induction of inhibitory surface molecules, including PD-1 [8].

So, where do COVID19 and cancer intersect?

The issue of carcinogenic effect of persistent SARS-CoV-2 infection was raised as early as Oct 2020 by Alpalhao and Filipe [9] who postulated tropism of the SARS-nCoV-2 virus towards immuneprivileged territories, where it can persist because of a failure of the cell to mount a $\mathrm{T}$ cell mediated immunity, thus kick-starting the pathogenesis of cancer by direct impairment of pRB and p53 tumour suppressor functions.

Vivarelli in Feb2021 [10] described the process of immune escape via a circRNA-microRNA-PD-1/PD-L1 axis, which reduces the cytotoxicity of NK cells. Antibodies which block the PD-1/PD-L1 pathway can restore T-cell competence, counteract viral infection and restore anti-tumoral immune response in cancer patients.

In May 2021, Zong., et al. [11] highlighted the clinical and molecular similarities between cancer and COVID-19 and summarized four major signaling pathways at the intersection of COVID-19 and cancer, namely, cytokine, IFN-I, AR and immune checkpoint signaling, but stopped short of directly implicating the SARS-CoV-2 virus in the pathogenesis of cancer.

When Yang et al analysed circRNAs in human lung epithelial cells infected with SARS-CoV-2, they found that the parental genes of circRNAs were involved in protective immune-inflammatory responses, and dysregulated circRNAs could regulate gene expression by absorbing the microRNAs, thus affecting mRNA stability, immunity, cell death [12].

\section{Let's wait for it}

Although several viral circRNAs, such as hpv-circE7, ebv-circRPMS1, and ebv-circLMP2A, are conclusively known to be associated with the development of cancers, there is a research gap with regard to circRNAs in coronaviruses, and there has been no isolation, identification or characterisation of a specific oncogenetic circRNA encoded by SARS-CoV-2.
Four clinical trials [10] are currently open, to examine the efficacy of antiPD1 antibody administration to both cancer and noncancer individuals affected by COVID19. The CAPTURE Study [7] whose recruitment started on $4^{\text {th }}$ May 2020, is a pan-cancer, longitudinal, immune profiling study, where among other things, the immunological interaction between the SARS-CoV-2, the host and cancer will be studied. We await with eagerness any further studies directed towards understanding the underlying molecular mechanisms that confirms the link between COVID-19 and cancer.

\section{Bibliography}

1. Cai Zena., et al. "Identification and characterization of circRNAs encoded by MERS-CoV, SARS-CoV-1 and SARS-CoV-2". Briefings in Bioinformatics 22.2 (2021): 1297-1308.

2. Cheng D., et al. "Cancer-related circular RNA: diverse biological functions". Cancer Cell International 21.11 (2021).

3. Lu M. "Circular RNA: functions, applications and prospects". ExRNA 2.1 (2020).

4. Kristensen L., et al. "Circular RNAs in cancer: opportunities and challenges in the field. Oncogene 37 (2018): 555-565.

5. Nahand Javid Sadri., et al. "Circular RNAs: New Epigenetic Signatures in Viral Infections". Frontiers in Microbiology 11 (2020): 1853.

6. Chen., et al. "N6-Methyladenosine Modification Controls Circular RNA Immunity”. Molecular Cell 76 (2019): 96-109.

7. Au Lewis., et al. "Cancer, COVID-19, and Antiviral Immunity: The CAPTURE Study". Cell 183.1 (2020): 4-10.

8. Quatrini Linda., et al. "The Immune Checkpoint PD-1 in Natural Killer Cells: Expression, Function and Targeting in Tumour Immunotherapy". Cancers 12.11 (2020): 3285.

9. Alpalhão Miguel., et al. "Persistent SARS-CoV-2 infection and the risk for cancer". Medical hypotheses 143 (2020): 109882.

10. Vivarelli S., et al. "Immune-checkpoint inhibitors from cancer to COVID19: A promising avenue for the treatment of patients with COVID19 (Review)". International Journal of Oncology 58.2 (2021): 145-157.

11. Zong Z., et al. "The intersection of COVID-19 and cancer: signaling pathways and treatment implications". Molecular Cancer 20.1 (2021): 76. 
12. Yang, Mengmei., et al. "Differential host circRNA expression profiles in human lung epithelial cells infected with SARSCoV-2". Infection, Genetics and Evolution: Journal of Molecular Epidemiology and Evolutionary Genetics in Infectious Diseases 93 (2021): 104923.

Volume 5 Issue 7 July 2021

(C) All rights are reserved by Sreelekha Ray. 